\title{
Pancreatic Carcinoma with Prominent Mucin Production: A Clinicopathologic and Immunohistochemical Study of 9 Cases
}

\author{
Makoto Saito $^{1}$, Hiroshi Nishihara ${ }^{2}$, Mishie Tanino ${ }^{1}$, Taichi Kimura ${ }^{1}$, Yoshihiro Matsuno ${ }^{3}$, \\ Kazuo Nagashima ${ }^{1,4}$, Michio Shimizu ${ }^{5}$, Shinya Tanaka ${ }^{{ }^{*}}$
}

${ }^{1}$ Department of Cancer Pathology, Hokkaido University Graduate School of Medicine, Sapporo, Japan; ${ }^{2}$ Department of Translational Pathology, Hokkaido University Graduate School of Medicine, Sapporo, Japan; ${ }^{3}$ Department of Surgical Pathology, Hokkaido University Hospital, Sapporo, Japan; ${ }^{4}$ Sapporo Higashi Tokushukai Hospital, Sapporo, Japan; ${ }^{5}$ Department of Pathology, Saitama Medical University, Saitama, Japan.

Email: *tanaka@med.hokudai.ac.jp

Received February $8^{\text {th }}, 2012$; revised March $1^{\text {st }}, 2012$; accepted March $21^{\text {st }}, 2012$

\begin{abstract}
Pancreatic carcinoma occasionally associated with prominent mucin production and this type of tumor designated as PCM (pancreatic carcinoma with prominent mucin production) was diagnosed depends on subjective estimation of the amount of mucous area, and there has been no report on a quantitative evaluation of the amount of mucinous area in the tumor. To examine the feature of PCM, we analyzed 9 cases of PCM among 243 cases of pancreas carcinoma and evaluated the amount of mucin by imaging analysis. Morphologically, 5 cases were classified as intradactal papillary mucinous neoplasms (IPMN)-derived PCM and 4 cases were as ductal adenocarcinoma (DA)-derived PCM. Mucous composition was found to be more than 50\% in all IPMN-derived PCM cases, and that was $40 \%-50 \%$ in DA-derived PCM cases with one exception. IPMN-derived PCM cases showed expansive growth with pancreatic duct dilatation filled with mucin, while DA-derived PCM cases possessed mucin infiltration into interstitial tissue. Immunohistochemically, three of 4 DA-derived PCM cases were MUC1(-)/MUC2(+), and the results of expressions for p16 and Dpc4 suggesting that DA-derived PCM was similar to IPMN-derived PCM rather than ordinary DA. Survival rate of DA-derived PCM cases was lower than that of IPMN-derived PCM cases. We advocate that DA-derived PCM may constitute a borderline group between IPMN and ordinary DA.
\end{abstract}

Keywords: Pancreatic Carcinoma with Prominent Mucin Production (PCM); Mucinous Non-Cystic Carcinoma (MNCC); Intraductal Papillary Mucinous Neoplasm (IPMN); Ductal Adenocarcinoma (DA); Immunohistochemistry

\section{Introduction}

Pancreatic carcinoma includes a certain population of those with prominent mucin production (PCM), including mucinous non-cystic carcinoma (MNCC), and few study have been reported because such cases were rare (1\% - 3\%) among invasive pancreatic carcinoma [1-3]. Recently, MNCC of the pancreas has been regarded as an advanced form of intraductal papillary mucinous neoplasm (IPMN) [4-6]. MNCC of the pancreas is defined in which mucin occupies more than $50 \%$ of tumor area by World Health Organization (WHO) [7], the Armed Forces Institute of Pathology (AFIP) [4], and the Japanese classification of pancreatic carcinoma [8]. However, the mucous area is subjectively estimated and no specific

*Corresponding author. methods have been reported to quantify the mucous area in PCM. In addition, few molecular biological evaluations of MNCC have been performed [9].

Pancreatic intraepithelial neoplasia (PanIN) is precursor lesion of ordinary ductal adenocarcinoma (DA), which is different from IPMN [10]. It has been shown that MUC1 expression suggests the "aggressive" pathway in which PanIN progressed to DA, while MUC2 is a marker for "indolent" pathway for IPMN-derived MNCC formation. The mucinous phenotype serves as a marker for the typing of pancreatic cancer [5,6]. In molecular models of pancreatic multistep carcinogenesis, the inactivation of the p16 gene, the overexpression of Cyclin D1, and the inactivation of the p53 and Dpc4 genes have been suggested as early, intermediate, and late events, respectively [11-15]. 
To analyze the clinicopathologic and immunohistochemical features of PCM, we analyzed 9 cases among 243 invasive pancreatic cancer cases. By the morphological analysis, we classified these 9 cases into two groups of PCM which derived from IPMN or DA, referred as IPMN-derived PCM and DA-derived PCM, respectively. The amount of mucin was measured by using imaging software. Immunostainings of MUC1/MUC2, p16, Cyclin D1, p53, and Dpc4 were performed. The clinicopathologic differences between IPMN- and DA-derived PCM were discussed.

\section{Materials and Methods}

\subsection{Case Analysis}

We reviewed 243 cases of pancreatic carcinoma which were surgically resected and diagnosed at the Laboratory of Cancer Research, Department of Pathology, Hokkaido University Graduate School of Medicine and the Department of Surgical Pathology, Hokkaido University Hospital from 1992 to 2003. For PCM lesions, 9 cases were selected in which conspicuous mucin was macroscopically seen diffusely over the tumor on cut surface. Cases with focal mucin or microscopically inside the cancer cells forming glandular lumens were excluded. Clinical stage was described based on pathological findings according to the Japanese classification [8].

PCM cases exhibiting intraductal papillary structure in dilated main and/or branch pancreatic duct with large amount of mucin, were regarded as being derived from IPMN $[4,7,8]$. All remaining PCM cases were regarded as being derived from DA. IPMN cases were further classified into intestinal type and pancreatobiliary type consisting of epithelial cells [16-18]. The protocol of this study conformed to the ethical guidelines of the Declaration of Helsinki and informed consent was obtained from each patients.

\subsection{Estimation of the Mucous Area (Figure 1)}

In all 9 PCM cases, the maximum cut surface of the tumor (1 - 9 slides) was almost in agreement with the site with the greatest mucin content. In lower magnification image of the H\&E staining, tumor lesions were discriminated using Adobe ${ }^{\circledR}$ Photoshop ${ }^{\circledR}$, and mucinous and non-mucinous parts were independently displayed with artificial colors as to avoid overlapping of either part (Figures 1(a) and (b)). The ratios in pixels of the mucinous part to the tumor lesion were obtained.

\subsection{Immunohistochemical Analysis}

The formalin-fixed, paraffin-embedded blocks obtained from the 9 PCM cases were performed for immunostain- ing with regular method described elsewhere (or site ref). Primary antibodies used are following: MUC1 (Clone Ma695, Novocastra Laboratories, Newcastle, UK; dilution 1:100), MUC2 (Clone Ccp 58, Novocastra Laboratories, Newcastle, UK; dilution 1:100), p16 (Clone ZJ11; NeoMarkers, Fremont, CA; dilution 1:50), Cyclin D1 (Clone SP4, Lab Vision, Fremont, CA; dilution 1:100), p53 (Clone DO-7, DAKO Corporation, Kyoto, Japan; dilution 1:500), and Dpc4 (Clone B8, Santa Cruz Biotechnology, Santa Cruz, CA; dilution 1:100).

Concerning MUC1, MUC2, p16, and Dpc4, the normal pancreatic tissue in each section served as an internal positive control. MUC1 and MUC2 were judged to be positive when $5 \%$ or more of the tumor cells were stained [16]. Cyclin D1 and p53 were judged to be overexpression when the nuclei of the tumor cells were homogeneously stained in $>5 \%$ and $>10 \%$, respectively [ 19 , 20]. Complete absence of nuclear expression of p16, or complete absence of cytoplasmic and nuclear expression of Dpc4, was judged to be negative [21,22]. Fifty ordinary DA cases were also examined as controls. Fisher's exact probability test was performed, and $\mathrm{p}<0.05$ was judged to be significant.

\section{Results}

\subsection{Clinicopathological Findings (Table 1)}

In 9 PCM cases, 5 were derived from IPMN, and the

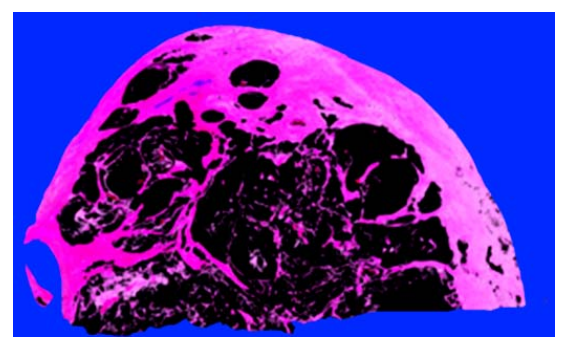

(a)

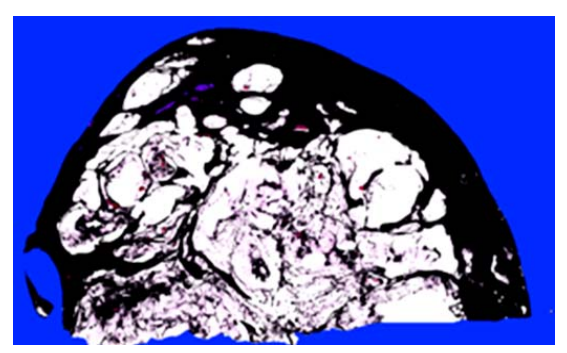

(b)

Figure 1. Representative calculation of the mucin rate of the tumor (Case 1). Mucinous (a) and non-mucinous (b) regions were manually evaluated as black pixels. In this section, the areas of the mucinous and non-mucinous regions were $2,416,649$ and $2,346,075$ pixels, respectively, and the mucin rate was $2,416,649 /(2,416,649+2,346,075) \times 100=50.7 \%$. 
Table 1. Clinicopathological findings of PCM cases.

\begin{tabular}{ccccccccc}
\hline \multicolumn{1}{c}{ Case } & Age & Sex & Location & Size & LN & pStage & Outcome & Mucin \\
\hline $\mathbf{1}$ & 71 & M & B-T & 5.6 & $(+)$ & IVa & Dead (17 M) & $58 \%$ \\
$\mathbf{2}$ & 75 & M & H & 8.4 & $(-)$ & III & Alive (67 M) & $64 \%$ \\
$\mathbf{3}$ & 54 & M & H & 11 & $(-)$ & IVa & Alive (48 M) & $61 \%$ \\
$\mathbf{4}$ & 56 & M & H & 7.2 & $(+)$ & IVa & Dead (6 M) & $60 \%$ \\
$\mathbf{5}$ & 70 & M & H & 3.3 & $(-)$ & III & Alive (69 M) & $54 \%$ \\
\hline & & & & DA-PCM & & & & \\
\hline $\mathbf{C a s e}$ & Age & Sex & Location & Size & LN & pStage & Outcome & Mucin \\
\hline $\mathbf{6}$ & 66 & M & T & 2.5 & $(+)$ & III & Dead (29 M) & $56 \%$ \\
$\mathbf{8}$ & 73 & M & H & 5.1 & $(+)$ & III & Dead (42 M) & $46 \%$ \\
$\mathbf{9}$ & 64 & M & T & 5.0 & $(+)$ & IVa & Dead (53 M) & $41 \%$ \\
\hline
\end{tabular}

remaining 4 from DA. In DA-derived PCM cases, 2 cases were located in the tail, and the mean maximum diameter was smaller than IPMN-derived PCM $(4.4 \mathrm{~cm}$ vs $7.1 \mathrm{~cm})$. All of DA-derived PCM cases had lymph node metastasis, and died after a mean postoperative period of 32.8 months. The 4 year survival rate of PCM derived from IPMN and DA were $60 \%$ and $0 \%$, respecttively.

\subsection{Mucin rate and Histopathologic Findings (Figure 2)}

The mucin rate was more than $40 \%$ in all PCM cases, and it was higher than 50\% (mean: 59.4\%) in all IPMNderived PCM. Five IPMN-derived PCM cases showed expansive growth with dilatation of pancreatic ducts filled with mucin (Figure 2(a)). Four cases were classified as intestinal type of IPMN (Figure 2(b)) and one case was recognized as pancreatobiliary type. In 4 DAderived PCM cases (Cases 6-9), the mucin rate was less than 50\% (mean: $45.8 \%$ ), excluding 1 case (56\%). The lesion of the exceptional one male case was clearly accompanied by cysts filling with mucin, but ovarian-type stroma as seen in mucinous cystic neoplasm was not present (Case 6). None of DA-derived PCM lesions showed gross dilatation of the pancreatic duct (Figure 2(c)). Histologically, all of DA-derived PCM cases were observed marked mucin infiltration into interstitial tissue and adenocarcinoma cells reside in a mucin pool (Figure 2(d)).

\subsection{Immunohistochemistry (Table 2 and Figure 3)}

For MUC1 and MUC2, 4 of 5 (80\%) IPMN (intestinal
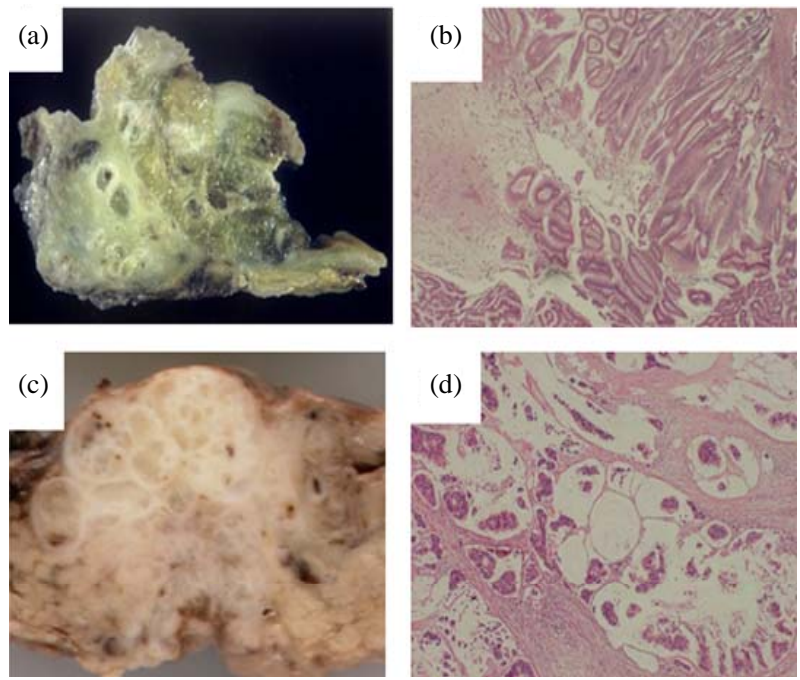

Figure 2. Representative features of PCM. (a) Macroscopic surface of IPMN-derived PCM (Case 2). Tumor filled with mucin expansively grew and directly invaded into the duodenal mucosa and the common bile duct; (b) Microscopic features of IPMN-derived PCM. Tumor cells were similar to colonic villous adenomas, and were classified as IPMN, intestinal type; (c) Macroscopic surface of DA-derived PCM (Case 7). Mucinous tumor was present in the head of the pancreas. Main pancreatic duct was unclear; (d) Microscopic features of DA-derived PCM. Conspicuous mucin was noted, and adenocarcinoma cells were floating in the mucin pool.

type)-derived PCM cases showed MUC1(-)/MUC2(+) except 1 IPMN (pancreatobiliary type)-derived PCM with MUC1(-)/MUC2(-). Among the 4 DA-derived PCM cases, 3 (75\%) were MUC1(-)/MUC2(+), while 1 was MUC1(+)/MUC2(-). In the 50 ordinary DA cases, 37 cases (74\%) showed MUC1(+) and all were MUC2(-). 
Table 2. Immunostaining of PCM cases comparison with ordinary ductal adenocarcinoma.

\begin{tabular}{cccc}
\hline & IPMN-PCM & DA-PCM & Ordinary DA \\
\hline MUC1 & $(0 / 5) 0 \%$ & $(1 / 4) 25 \%$ & $(37 / 50) 100 \%$ \\
MUC2 & $(4 / 5) 80 \%$ & $(3 / 4) 75 \%$ & $(0 / 50) 0 \%$ \\
p16 & $(5 / 5) 100 \%$ & $(3 / 4) 75 \%$ & $(13 / 50) 26 \%$ \\
CyclinD1 & $(1 / 5) 20 \%$ & $(1 / 4) 25 \%$ & $(22 / 50) 44 \%$ \\
p53 & $(2 / 5) 40 \%$ & $(1 / 4) 25 \%$ & $(23 / 50) 46 \%$ \\
Dpc4 & $(4 / 5) 80 \%$ & $(3 / 4) 75 \%$ & $(20 / 50) 40 \%$ \\
\hline
\end{tabular}

Not only IPMN- but also DA-derived PCM showed significant positivity for MUC2 which is different from ordinary DA.

For p16 (Figure 3(a)), Cyclin D1 (Figure 3(b)), p53 (Figure 3(c)), and Dpc4 (Figure 3(d)), positivity for p16 and Dpc4 tended to be higher in PCM derived either from IPMN or DA than those seen in ordinary DA. Overexpression of Cyclin D1 and p53 did not differ between PCM, derived either from IPMN or DA, and ordinary DA.

\section{Discussion}

The mucin contents of pancreas carcinoma have been evaluated by diverse criteria. Suda et al. suggested the importance of macroscopical observation as abundant mucin or muco-nodules, and of presence of floating cancer cells in mucin lakes for diagnosis of MNCC [1]. Adsay et al. described that percentage mucin is $80 \%$ or higher to for MNCC distinguished from IPMN [2]. Generally, MNCC has been defined as that mucinous part occupying more than $50 \%$ of the tumor $[3,4,7,8]$. However, the evaluation of the mucous area has been made subjectively. For the evaluation of PCM, in this study, imaging software was employed, and we objectively determined the percentage of the mucinous component. In our study, 5 out of 9 cases (56\%) of PCM arose from IPMN, in consistent with the previous report describing that $73 \%$ or $53 \%$ of mucin rich pancreas carcinoma was derived from IPMN by Suda et al. [1], or Adsay et al. [2], respectively. Reflecting on the histological features of IPMN-derived PCM which tends to grow expansively, mucin composition was higher than DA-derived PCM. With $50 \%$ as a borderline, PCM could be classified according to its derivation based on the mucin rate into DA-derived $(40 \%-50 \%)$ and IPMN-derived $(\geq 50 \%)$ PCM (Figure 4).

Marked mucin infiltration into interstitial tissue was observed all of DA-derived PCM cases. While all of 50 ordinary DA cases were MUC2(-), 3 of 4 DA-derived PCM cases wre MUC2(+). In DA-derived PCM cases,
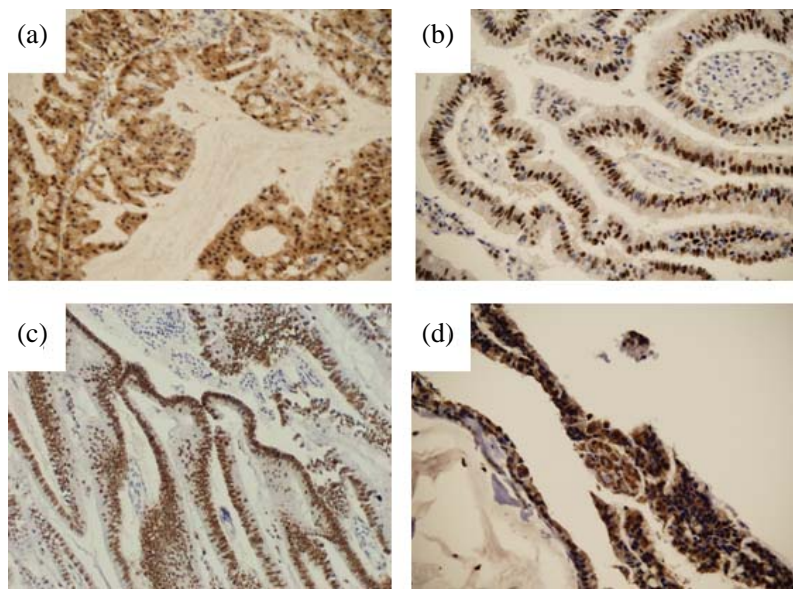

Figure 3. Representative results of immunostaining. (a) p16 in Case 3; (b) Cyclin D1 in Case 5; (c) p53 in Case 2, and (d) Dpc4 in Case 1.

PCM: Pancreatic carcinoma with prominent mucin production

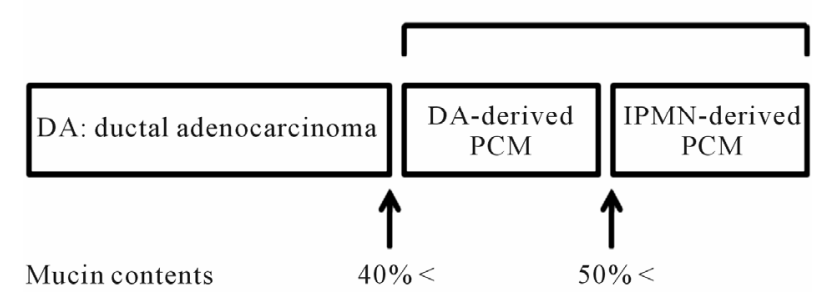

Figure 4. Correlation of the mucin contents and histological subtype of pancreatic cancer.

the expression patterns of p16 and Dpc4 were similar to those of IPMN-derived PCM rather than ordinary DA. Thus, despite the mucin rate was less than 50\%, DAderived PCM seemed to be included in MNCC. However, the cases in DA-derived PCM exhibited a worse 4-year survival rate compared with IPMN-derived PCM. Therefore, we propose a hypothesis that DA-derived PCM constitutes a borderline group between IPMN and ordinary DA. Further studies are required to clarify the biological relevance of DA-derived PCM.

In conclusion, we quantitate the mucinous area of PCM. PCM should have more than $40 \%$ of mucous area and especially IPMN-derived PCM contain the mucous area more than 50\%. DA-derived PCM was similar to IPMNderived PCM immunophenotypically rather than ordinary DA. This study provides the prototype of further analysis of PCM by evaluating large numbers of cases in the future.

\section{Acknowledgements}

We thank Fumihiro Sata, PhD (Department of Public Health, Hokkaido University Graduate School of Medicine) for expert support of statistical analysis. We thank 
Dr. Hirofumi Kamachi (Hokkaido University Hospital) and Dr. Satoshi Hirano (Hokkaido University Hospital) for preparing clinical information, and Katsuji Marukawa for excellent technical assistance. This study was supported in part by Grants-in-Aid from the Ministry of Education, Science, Culture, and Sports, and from the Ministry of Health, Labor, and Welfare; and also by the YASUDA Medical Research Foundation, by the SUHARA Memorial Foundation, by the Mochida Memorial Foundation for Medical and Pharmaceutical Research, and by the UEHARA Medical Research Foundation.

\section{REFERENCES}

[1] K. Suda, B. Nobukawa, S. Yamasaki, F. Suzuki, H. Shimizu and M. Takase, "Pre-Existing Histological Type and Developmental Mechanism of Mucinous Noncystic Carcinoma of Pancreas," Pathology \& Oncology Research, Vol. 6, No. 2, 2000, pp. 125-129. doi:10.1007/BF03032362

[2] N. V. Adsay, C. Pierson, F. Sarkar, J. Abrams, D. Weaver, K. C. Conlon, M. F. Brennan and D. S. Klimstra, "Colloid (Mucinous Noncystic) Carcinoma of the Pancreas," American Journal of Surgical Pathology, Vol. 25, No. 1, 2001, pp. 26-42.

doi:10.1097/00000478-200101000-00003

[3] G. Seidel, M. Zahurak, C. Iacobuzio-Donahue, T. A. Sohn, N. V. Adsay, C. J. Yeo, K. D. Lillemoe, J. L. Caeron, R. H. Hruban and R. E. Wilentz, "Almost All Infiltrating Colloid Carcinomas of the Pancreas and Periampullary Region Arise from in Situ Papillary Neoplasms: A Study of 39 Cases," American Journal of Surgical Pathology, Vol. 26, No. 1, 2002, pp. 56-63. doi:10.1097/00000478-200201000-00006

[4] R. H. Hruban, M. B. Pitman and D. S. Klimstra, "Tumors of the Pancreas, Afip Atlas of Tumor Pathology, 4th Series Fascicle 6," 6th Edition, American Registry of Pathology, Washington DC, 2007.

[5] J. Lüttges, G. Zamboni, D. Longnecker and G. Klöppel, "The Immunohistochemical Mucin Expression Pattern Distinguishes Different Types of Intraductal Papillary Mucinous Neoplasms of the Pancreas and Determines Their Relationship to Mucinous Noncystic Carcinoma and Ductal Adenocarcinoma," American Journal of Surgical Pathology, Vol. 25, No. 7, 2001, pp. 942-948. doi:10.1097/00000478-200107000-00014

[6] N. V. Adsay, K. Merati, A. Andea, F. Sarkar, R. H. Hruban, R. E. Wilentz, M. Goggins, C. IocobuzioDonahue, D. S. Longnecker and D. S. Klimstra, "The Dichotomy in the Preinvasive Neoplasia to Invasive Carcinoma Sequence in the Pancreas: Differential Expression of MUC1 and MUC2 Supports the Existence of Two Separate Pathways of Carcinogenesis," Modern Pathology, Vol. 15, No. 10, 2002, pp. 1087-1095. doi:10.1097/01.MP.0000028647.98725.8B

[7] S. R. Hamilton and L. A. Aaltonen, "World Health Organization Classification of Tumours; Pathology \& Ge- netics, Tumours of the Digestive System,” IARC Press, Lyon, 2000.

[8] Y. Kawarada, "Classification of Pancreatic Carcinoma," 2nd Edition, Japan Pancreas Society, Tokyo, 2003.

[9] J. Lüttges, K. Beyser, S. Pust, A. Paulus, J. Ruschoff and G. Klöppel, "Pancreatic Mucinous Noncystic (Colloid) Carcinomas and Intraductal Papillary Mucinous Carcinomas Are Usually Microsatellite Stable,” Modern Pathology, Vol. 16, No. 6, 2003, pp. 537-342. doi:10.1097/01.MP.0000072748.65178.2F

[10] R. H. Hruban, N. V. Adsay, J. Albores-Saavedra, C. Compton, E. S. Garrett, S. N. Goodman, S. E. Kern, D. S. Klimstra, G. Klöppel, D. S. Longnecker, J. Lüttges and G. J. Offerhaus, "Pancreatic Intraepithelial Neoplasia: A New Nomenclature and Classification System for Pancreatic Duct Lesions,” American Journal of Surgical Pathology, Vol. 25, No. 5, 2001, pp. 579-586. doi:10.1097/00000478-200105000-00003

[11] A. Maitra, N. V. Adsay, P. Argani, C. Iacobuzio-Donahue, A. De Marzo, J. L. Cameron, C. J. Yeo and R. H. Hruban, "Multicomponent Analysis of the Pancreatic Adenocarcinoma Progression Model Using a Pancreatic Intraepithelial Neoplasia Tissue Microarray,” Modern Pathology, Vol. 16, No. 9, 2003, pp. 902-912. doi:10.1097/01.MP.0000086072.56290.FB

[12] A. V. Biankin, J. G. Kench, A. L. Morey, C. S. Lee, S. A. Biankin, D. R. Head, T. B. Hugh, S. M. Henshall and R. L. Sutherland, "Overexpression of p21(WAF1/CIP1) Is an Early Event in the Development of Pancreatic Intraepithelial Neoplasia,” Cancer Research, Vol. 61, No. 24, 2001, pp. 8830-8837.

[13] A. V. Biankin, S. A. Biankin, J. G. Kench, A. L. Morey, C. S. Lee, D. R. Head, R. P. Eckstein, T. B. Hugh, S. M. Henshall and R. L. Sutherland, "Aberrant p16 (Ink4a) and Dpc4/Smad4 Expression in Intraductal Papillary Mucinous Tumours of the Pancreas Is Associated with Invasive Ductal Adenocarcinoma,” Gut, Vol. 50, No. 6, 2002, pp. 861-868.

[14] R. H. Hruban, K. Takaori, D. S. Klimstra, N. V. Adsay, J. Albores-Saavedra, A. V. Biankin, S. A. Biankin, C. Compton, N. Fukushima, T. Furukawa, M. Goggins, Y. Kato, G. Klöppel, D. S. Longnecker, J. Lüttges, A. Maitra, G. J. Offerhaus, M. Shimizu and S. Yonezawa, “An Illustrated Consensus on the Classification of Pancreatic Intraepithelial Neoplasia and Intraductal Papillary Mucinous Neoplasms," American Journal of Surgical Pathology, Vol. 28, No. 8, 2004, pp. 977-987. doi:10.1097/01.pas.0000126675.59108.80

[15] A. V. Biankin, J. G. Kench, S. A. Biankin, C. S. Lee, A. L. Morey, F. P. Dijkman, M. J. Coleman, R. L. Sutherland and S. M. Henshall, "Pancreatic Intraepithelial Neoplasia in Association with Intraductal Papillary Mucinous Neoplasms of the Pancreas: Implications for Disease Progression and Recurrence," American Journal of Surgical Pathology, Vol. 28, No. 9, 2004, pp. 1184-1192. doi:10.1097/01.pas.0000131556.22382.3c

[16] A. Nakamura, M. Horinouchi, M. Goto, K. Nagata, K. Sakoda, S. Takao, K. Imai, Y. S. Kim, E. Sato and S. 
Yonezawa, "New Classification of Pancreatic Intraductal Papillary-Mucinous Tumour by Mucin Expression: Its Relationship with Potential for Malignancy," Journal of Pathology, Vol. 197, No. 2, 2002, pp. 201-210. doi:10.1002/path.1109

[17] N. V. Adsay, K. Merati, O. Basturk, C. IacobuzioDonahue, E. Levi, J. D. Cheng, F. H. Sarkar, R. H. Hruban and D. S. Klimstra, "Pathologically and Biologically Distinct Types of Epithelium in Intraductal Papillary Mucinous Neoplasms: Delineation of an 'Intestinal' Pathway of Carcinogenesis in the Pancreas," American Journal of Surgical Pathology, Vol. 28, No. 7, 2004, pp. 839848. doi:10.1097/00000478-200407000-00001

[18] T. Furukawa, G. Klöppel, N. Volkan Adsay, J. AlboresSaavedra, N. Fukushima, A. Horii, R. H. Hruban, Y. Kato, D. S. Klimstra, D. S. Longnecker, J. Lüttges, G. J. Offerhaus, M. Shimizu, M. Sunamura, A. Suriawinata, K. Takaori and S. Yonezawa, "Classification of Types of Intraductal Papillary-Mucinous Neoplasm of the Pancreas: A Consensus Study,” Virchows Archiv, Vol. 447, No. 5, 2005, pp. 794-799. doi:10.1007/s00428-005-0039-7
[19] K. M. Alle, S. M. Henshall, A. S. Field and R. L. Sutherland, "Cyclin D1 Protein Is Overexpressed in Hyperplasia and Intraductal Carcinoma of the Breast," Clinical Cancer Research, Vol. 4, No. 4, 1998, pp. 847-854.

[20] R. J. Bova, D. I. Quinn, J. S. Nankervis, I. E. Cole, B. F. Sheridan, M. J. Jensen, G. J. Morgan, C. J. Hughes and R. L. Sutherland, "Cyclin D1 and p16 ${ }^{\text {INK4A }}$ Expression Predict Reduced Survival in Carcinoma of the Anterior Tongue,” Clinical Cancer Research, Vol. 5, No. 10, 1999, pp. 2810-2819.

[21] R. E. Wilentz, J. Geradts, R. Maynard, G. J. Offerhaus, M. Kang, M. Goggins, C. J. Yeo, S. E. Kern and R. H. Hruban, "Inactivation of the p16 (Ink4a) Tumor-Suppressor Gene in Pancreatic Duct Lesions: Loss of Intranuclear Expression,” Cancer Research, Vol. 58, No. 20, 1998, pp. 4740-4744.

[22] K. M. Alle, S. M. Henshall, A. S. Field and R. L. Sutherland, "Cyclin D1 Protein Is Overexpressed in Hyperplasia and Intraductal Carcinoma of the Breast," Clinical Cancer Research, Vol. 4, No. 4, 1998, pp. 847-854. 